\title{
Mangas das variedades espada, rosa e tommy atkins: compostos bioativos e potencial antioxidante
}

\section{Mango varieties "espada", "rosa" and tommy atkins: bioactive compounds and antioxidant potential}

\author{
Enayde de Almeida Melor*; Cristiane Rodrigues de Araújo²
}

Resumo

O objetivo deste trabalho foi quantificar o teor de ácido ascórbico, carotenóides e fenólicos totais e avaliar o potencial antioxidante de mangas das variedades Espada, Rosa e Tommy Atkins. As frutas foram desintegradas para obtenção da polpa, os resíduos resultantes desidratados, triturados e tamisados. Extratos hidroacetônico, hidrometanólico e aquoso, obtidos por processo de extração sequencial a partir da polpa fresca e do resíduo desidratado foram submetidos à determinação da capacidade de seqüestro do radical DPPH. A manga Espada e a Tommy Atkins apresentaram teor de carotenóides totais semelhante entre si, porém inferior ao da manga Rosa. A manga Tommy Atkins exibiu o menor teor de ácido ascórbico e a Espada o maior teor de fenólicos totais. O extrato hidroacetônico da polpa e do resíduo exibiu uma forte capacidade de seqüestro (superior a $80 \%$ ).

Palavras-chave: Antioxidante, Mangifera indica L., fitoquímicos bioativos

\begin{abstract}
The aim of this work was to determine the amount of ascorbic acid, total phenolics and carotenoids and evaluate the antioxidant potential of mango varieties "Espada", "Rosa" and Tommy Atkins. The fruits were ground to obtain the fresh pulp and waste resulting was dried, crushed to pass through sieve. Hydroacetone, hydromethanolic and aqueous extracts, obtained through sequential extraction procedure from pulp and dehydrated waste were screened for their antioxidant activity by DPPH free radical scavenging. The "espada" and Tommy Atkins mango showed similar levels of total carotenoids, however less than Rosa mango. The Tommy Atkins mango exhibited the lowest content of ascorbic acid and the espada mango the highest level of phenolic compounds. The extract hydroacetone (pulp and dehydrated waste) exhibited a strong free radical scavenging capacity ( $>80 \%$ ).
\end{abstract}

Key words: Antioxidant, Mangifera indica L., phytochemical bioativos

\section{Introdução}

A manga (Mangifera indica L.) é uma fruta de aroma e cor agradáveis, pertencente à família Anacardiaceae, que integra o elenco das frutas tropicais de importância econômica (BRANDÃO;
MAIA; LIMA, 2003). Em termos de produção mundial e de área cultivada é a segunda fruta mais importante, depois da banana (BERNADESSILVA; LAJOLO; CORDENUNSI, 2003). O Brasil, com uma produção anual de 1.272.184 toneladas, no ano de 2007, da qual 970.786 toneladas foram

\footnotetext{
1 Doutora em Nutrição, Prof ${ }^{\mathrm{a}}$ Associado II da Universidade Federal Rural de Pernambuco, UFRPE. Prof ${ }^{\mathrm{a}}$ permanente do Programa de Pós-graduação em Ciência e Tecnologia de Alimentos da UFRPE. E-mail: eamelo@dcd.ufrpe.br

2 Graduada em Economia Doméstica pela UFRPE, Bolsista PIBIC/CNPq-UFRPE por 02 anos; mestranda do Programa de Ciência e Tecnologia de Alimentos da UFRPE. E-mail: cristiane_rodriguespenna@yahoo.com.br

* Autor para correspondência
} 
produzidas na Região Nordeste, ocupa posição de destaque entre os principais países em função do volume de produção e exportação (IBGE, 2009).

Estima-se existir em solo brasileiro, um elevado número de variedades conhecidas de mangas. Algumas dessas variedades são originárias do velho mundo, outras são oriundas de cruzamentos aleatórios nos campos, ou foram geneticamente induzidas, buscando a melhoria de variedades de alto valor agregado (GOMES, 1983). Dentre as variedades introduzidas no Brasil a partir da década de 60 que constituem a base da mangicultura brasileira para exportação, destaca-se a Tommy Atkins. Esta variedade juntamente com a variedade Rosa e Espada são as de maior produção e consumo na região Nordeste brasileira (GENÚ; PINTO, 2002).

No mercado interno, mangas são comercializadas e consumidas na forma in natura, no entanto, como acontecem com outras frutas, os produtos industrializados vem despertando o interesse do consumidor. Desta forma, a polpa de manga constitui matéria-prima de importância para as indústrias de conservas de frutas, que podem produzi-las durante as épocas de safra, armazená-las e reprocessá-las em períodos mais propícios, ou segundo a demanda do mercado consumidor, para obtenção de doces em massa, geléias, sucos e néctares. Além disso, outras indústrias alimentícias adquirem a polpa de manga para empregá-la em formulação de iogurtes, doces, biscoitos, bolos, sorvetes, refrescos e alimentos infantis (BENEVIDES et al., 2008).

A manga, além de ser uma fruta bastante apreciada, constitui uma importante fonte de fitoquímicos, dentre os quais se destacam os polifenóis, os carotenóides e a vitamina C (KIM; BRECHT; TALCOTT, 2007; FRANKE et al., 2004; GODOY; RODRIGUEZ-AMAYA, 1989). Estes fitoquímicos por exibirem propriedade antioxidante atuam retardando a velocidade da reação de oxidação, por ação sinérgica ou não, protegendo o organismo contra os radicais livres
(KAUR; KAPOOR, 2001). Vale destacar que o teor destes compostos em vegetais varia, entre outros fatores, em função das condições edafoclimáticas do cultivo, variedade e grau de maturação da fruta (SCALZO et al., 2005). Vale ressaltar, ainda, que algumas frutas podem potencialmente conter maior teor de compostos antioxidantes nas sementes e cascas do que na polpa, ou ainda, o perfil dos fitoquímicos antioxidantes ser diferenciado nestas partes do vegetal (GUO et al., 2003). Como consequência, muitas vezes, a ação antioxidante das sementes e cascas é superior à exibida pala porção comestível da fruta, como evidenciado por Soong e Barlow (2004) ao estudar a ação antioxidante das sementes e porção comestível de abacate, jaca, manga e tamarindo. Estas constatações motivaram o desenvolvimento deste trabalho com vista a avaliar o potencial antioxidante de mangas das variedades espada, rosa e Tommy Atkins comercializadas na Cidade do Recife - PE.

\section{Material e Métodos}

Mangas das variedades espada, rosa e Tommy Atkins, no estádio de maturação maduro, adquiridas no comércio local, foram analisados, quanto ao teor de fenólicos totais, ácido ascórbico e carotenóides totais e sua ação antioxidante.

\section{Preparo das amostras}

a) As mangas foram higienizadas, fatiadas e transformadas em polpa, utilizando uma centrifuga doméstica (Wallita) as quais foram submetidas às determinações analíticas abaixo descritas.

b) Os resíduos resultantes da obtenção da polpa fresca de manga, com exceção do caroço, foram secos em estufa com circulação forçada $\left(\mathrm{a} 40^{\circ} \mathrm{C}\right)$, até atingir a umidade inferior a 10\%, que foi mensurada, por pesagem, em balança infravermelha. Em sequência, os resíduos secos foram triturados em moinho, e peneirados para obtenção de uma farinha de granulometria uniforme. 


\section{Obtenção dos extratos}

Os extratos hidroacetônico, hidrometanólico e aquoso da polpa e do resíduo desidratado das mangas foram obtidos por processo de extração sequencial, a saber: $30 \mathrm{~g}$ da polpa e $20 \mathrm{~g}$ do resíduo desidratado foram mantidos sob agitação permanente, em temperatura ambiente $\left(24^{\circ} \mathrm{C} \pm 2^{\circ} \mathrm{C}\right)$, por 20 minutos, em acetona a $80 \%$, na proporção de $1: 2$ $(\mathrm{p} / \mathrm{v})$, e em seguida, filtrada. O resíduo resultante da filtração foi ressuspenso em acetona a $80 \%$, e novamente submetido ao processo de extração por mais dois períodos de 20 minutos, totalizando 60 minutos de extração. $\mathrm{O}$ extrato resultante foi combinado, concentrado sob pressão reduzida a $40^{\circ}$ $\mathrm{C}$, e o volume final aferido para $50 \mathrm{~mL}$. O resíduo resultante da filtração foi reutilizado para a extração com metanol a $80 \%$ e, subseqüentemente, com água destilada nas mesmas condições acima descritas. Os extratos obtidos foram acondicionados em vidros âmbar, fechados e mantidos sob congelamento $\left(-18^{\circ} \mathrm{C}\right)$ até o momento das análises.

\section{Determinações analíticas}

\section{Fenólicos totais}

O teor de fenólicos totais foi determinado por método espectrofotométrico, utilizando o reagente Folin-Ciocalteau (Merck), segundo metodologia descrita por Wettansighe e Shahidi (1999) e curva padrão de catequina. Os resultados foram expressos em mg de fenólicos totais em equivalente de catequina por grama da amostra e em $\mu \mathrm{g}$ em equivalente de catequina por $\mathrm{mL}$ do extrato.

\section{Carotenóides totais}

A extração dos carotenóides foi efetuada pelo método descrito por Rodriguez-Amaya (1999), utilizando $5 \mathrm{~g}$ da polpa fresca e $1 \mathrm{~g}$ da farinha dos resíduos de manga. Para sua quantificação foi utilizado o espectro de absorção (absorbância), registrado no comprimento de onda de $450 \mathrm{~nm}$, considerando o coeficiente de absorção ( $E^{1 \%}$ cm) de 2500 e a seguinte expressão matemática: $\mu \mathrm{g} / \mathrm{g}=\left(\right.$ Volume $\mathrm{x}$ Absorbância X 106) $/ \mathrm{E}^{1 \%}{ }_{\mathrm{cm}} \mathrm{x}$ peso da $\operatorname{amostra}(\mathrm{g}) \times 100$. Todas as etapas desta determinação foram conduzidas ao abrigo da luz e os resultados expressos em $\mu \mathrm{g}$ de carotenóides totais em equivalente de $\beta$-caroteno por grama da amostra.

\section{Ácido ascórbico}

Uma alíquota de $2 \mathrm{~g}$ da polpa e $1 \mathrm{~g}$ da farinha dos resíduos de manga foi submetida à determinação do teor de ácido ascórbico, por método titulométrico, utilizando 2,6 diclofenol indofenol (AOAC, 1990).

\section{Ensaio da atividade antioxidante}

A atividade antioxidante do extrato hidroacetônico, hidrometanólico e aquoso da polpa e dos resíduos desidratados de manga foi determinada, isoladamente, utilizando o método da capacidade de seqüestrar o radical 1,1-difenil-2picrihidrazil (DPPH), descrito por Brand-Williams, Cuvelier e Berset (1995). Alíquotas dos extratos foram colocadas em diferentes tubos de ensaio, e adicionadas da solução de DPPH em metanol $(0,1 \mathrm{M})$, atingindo volume final de $6 \mathrm{~mL}$. Em seguida, os tubos foram deixados em repouso, ao abrigo da luz, e ao final de 15, 30, 45 e 60 minutos a absorbância foi medida a $515 \mathrm{~nm}$. A capacidade de seqüestrar o radical, expressa em percentual, foi calculada em relação ao controle (sem antioxidante), segundo a expressão descrita por Miliauskas, Venskutonis e Van Beek (2004): \% seqüestro $=($ Absorbância do controle - Absorbância da amostra / Absorbância do controle) x 100 .

Como termo de comparação foi utilizado à capacidade de seqüestrar o radical DPPH do antioxidante sintético BHT (butil-hidroxitolueno) e do ácido ascórbico, determinada nas mesmas condições dos ensaios acima descritos. 


\section{Análise estatística}

Todas as determinações foram efetuadas em triplicata e os resultados submetidos à análise de variância e teste de Duncan ao nível de 5\% de probabilidade, utilizando o programa estatístico "Statistic - for Windows" versão 6.

\section{Resultados e Discussão}

Fitoquímicos em mangas das variedades Espada, Rosa e Tommy Atkins e a capacidade de seqüestrar o radical 1,1-difenil-2-picrihidrazil (DPPH) de seus extratos

O teor dos principais fitoquímicos antioxidantes presentes em três variedades de manga encontrase na Tabela 1. A manga Espada e a Tommy Atkins apresentaram teor de carotenóides totais, estatisticamente semelhantes, porém significativamente inferior ao da manga Rosa. O teor destes fitoquímicos foi superior ao encontrado por Padilha (2005) em manga Espada $(12,12 \mu \mathrm{g}$. $\left.\mathrm{g}^{-1}\right)$ e Rosa $\left(27,06 \mu \mathrm{g} . \mathrm{g}^{-1}\right)$, e inferior ao da Tommy Atkins $\left(34,45 \mu \mathrm{g}\right.$. $\left.\mathrm{g}^{-1}\right)$. Em manga Espada e Rosa comercializada na cidade do Recife-PE, Melo et al. (2006) relatam valores de 17,98 $\mu \mathrm{g}$. $\mathrm{g}^{-1}$ e de $24,98 \mu \mathrm{g}$. $\mathrm{g}^{-1}$, respectivamente. As discrepâncias entre as variedades possivelmente decorre do perfil diferenciado de carotenóides apresentado pelas mesmas, além disso, as condições edafoclimáticas do cultivo e o grau de maturação dos frutos que influenciam o teor destes fotoquímicos em vegetais (CHEN; TAI; CHEN, 2004) podem justificar as diferenças entre as frutas da mesma variedade. Mercadante (1994) refere um conteúdo de carotenóides para mangas Tommy Atkins na faixa de 10,6 a $25,8 \mu \mathrm{g} \cdot \mathrm{g}^{-1}$ na fruta verde; 23,7 a 65,1 $\mu \mathrm{g} . \mathrm{g}^{-1}$ na fruta parcialmente madura e 40,1 a 70,5 $\mu \mathrm{g} \cdot \mathrm{g}^{-1}$ na fruta madura.

Tabela 1. Teor de fitoquímicos em manga Espada, Rosa e Tommy Atkins.

\begin{tabular}{llcc}
\hline Fitoquímicos & \multicolumn{1}{c}{ Manga (Variedades) } & \\
& Espada & Rosa & Tommy Atkins \\
\hline Carotenóides totais* $\left(\mu \cdot \mathrm{g}^{-1}\right)$ & $29,57 \pm 1,95 \mathrm{~b}$ & $48,06 \pm 2,98 \mathrm{a}$ & $28,13 \pm 1,82 \mathrm{~b}$ \\
Ácido ascórbico $\left(\mathrm{mg} \cdot 100 \mathrm{~g}^{-1}\right)$ & $38,21 \pm 2,81 \mathrm{a}$ & $28,70 \pm 1,83 \mathrm{ab}$ & $20,85 \pm 1,72 \mathrm{~b}$ \\
Fenólicos totais** $\left(\mathrm{mg} .100 \mathrm{~g}^{-1}\right)$ & $279,54 \pm 17,01 \mathrm{a}$ & $126,08 \pm 9,45 \mathrm{~b}$ & $133,23 \pm 8,23 \mathrm{~b}$ \\
\hline
\end{tabular}

Médias de três determinações independentes. Médias seguidas por letras iguais na linha não diferem estatisticamente pelo Teste de Duncan $(\mathrm{p}<0,05) .{ }^{*} \mu \mathrm{g}$ em equivalente de $\beta$-caroteno. $\mathrm{g}^{-1}$ de amostra; $* *$ mg em equivalente de catequina. $100 \mathrm{~g}^{-1}$

No que se refere ao teor de ácido ascórbico evidencia-se que a manga Tommy Atkins exibiu, estatisticamente, o menor valor quando comparada com o da manga Espada, porém semelhante ao da manga Rosa. Bezerra et al. (2008) detectaram em manga Rosa teor de ácido ascórbico de 60,80 mg.100g-1, enquanto que Melo et al. (2006) fazem referência ao teor de 57,65 e $83,90 \mathrm{mg} \cdot 100 \mathrm{~g}^{-1}$ de ácido ascórbico para a manga Espada e Rosa, respectivamente. A manga Espada, também exibiu o maior teor de fenólicos totais seguida da Tommy Atkins e Rosa. Padilha (2005) utilizando metanol a $80 \%$ como solvente extrator relata teor de fenólicos totais de 20,02; 54,76 e 24,34 mg 100 $\mathrm{g}^{-1}$ em manga Espada, Rosa e Tommy Atkins, respectivamente, valores inferiores aos obtidos neste estudo.

A maioria dos polifenóis presentes na fruta, independente da variedade, foi solubilizada em acetona a $80 \%$, enquanto que quantidades menores 
foram solubilizadas, subseqüentemente, em metanol $80 \%$ e água. O extrato aquoso da manga Rosa apresentou maior teor de polifenóis do que o extrato hidrometanólico (Tabela 2). Verifica-se que a utilização sequencial de solventes favoreceu a extração destes fitoquímicos. Concordando, assim, com Pérez-Jiménez et al. (2008) que ressaltam que, para a eficiência do processo de extração, devese combinar pelo menos dois ciclos de extração, utilizando-se soluções de solventes orgânicos aquosos, com diferentes polaridades, de modo a extrair compostos com diferentes estruturas químicas. Estes resultados demonstramanecessidade de efetuar seleção criteriosa do solvente de extração, tendo em vista que em vegetais existem polifenóis com polaridade diversificada, e que a solubilidade destes constituintes em um determinado solvente é uma característica peculiar do fitoquímico. Assim, a eficácia do solvente dependerá da polaridade dos polifenóis presentes na amostra, bem como do grau de polimerização e da interação com outros constituintes (NACZK; SHAHIDI, 2004).

Tabela 2. Fenólicos totais* em extratos de manga Espada, Rosa e Tommy Atkins.

\begin{tabular}{lcccc}
\hline Manga & \multicolumn{3}{c}{ Extratos } \\
\cline { 2 - 5 } (variedades) & Hidroacetônico & Hidrometanólico & Aquoso & Total \\
\hline Espada & $1.561,98^{\mathrm{a}}$ & $95,42^{\mathrm{a}}$ & $19,93^{\mathrm{b}}$ & $1.677,33^{\mathrm{a}}$ \\
Rosa & $652,85^{\mathrm{b}}$ & $42,56^{\mathrm{b}}$ & $60,97^{\mathrm{a}}$ & $756,38^{\mathrm{b}}$ \\
Tommy Atkins & $740,31^{\mathrm{b}}$ & $41,54^{\mathrm{b}}$ & $22,58^{\mathrm{b}}$ & $804,43^{\mathrm{b}}$ \\
\hline
\end{tabular}

Médias de três determinações. Médias seguidas por letras iguais na coluna não difere de forma estatisticamente pelo Teste de Duncan $(\mathrm{p}<0,05) .{ }^{*} \mu \mathrm{g}$ em equivalente de catequina. $\mathrm{mL}^{-1}$ de extrato.

Os extratos ao serem submetidos ao ensaio da atividade antioxidante exibiram ação diferenciada. O extrato hidroacetônico, com maior teor de polifenóis, exibiu a mais elevada capacidade de seqüestro do radical DPPH, superior a $80 \%$, independente da variedade de manga (Figura 1). A ação antioxidante deste extrato foi estatisticamente semelhante a do ácido ascórbico e inferior ao BHT. Os extratos hidrometanólicos da manga Tommy Atkins e Rosa exibiram capacidade de seqüestro semelhante, porém inferior a do extrato hidrometanólico da manga Espada. A mais baixa ação antioxidante foi exibida pelos extratos aquosos de todas as mangas (inferior a 10\%), não havendo diferença estatística entre elas. No entanto, ao misturar os extratos, na proporção de 1:1:1 (v/v/v), a ação antioxidante atingiu valores superiores a $80 \%$, com destaque para o extrato de manga Espada e Tommy Atkins que exibiu ação antioxidante semelhante a do ácido ascórbico e do BHT desde o inicio da reação.

Fitoquímicos em resíduo desidratado de manga das variedades Espada, Rosa e Tommy Atkins a capacidade de seqüestrar o radical 1,1-difenil-2-picrihidrazil (DPPH) de seus extratos

O resíduo desidratado, resultante da obtenção da polpa fresca de manga de três variedades, ainda apresentou teor relevante de fitoquímicos. $\mathrm{O}$ resíduo da manga Espada exibiu o maior teor de fenólicos totais, seguido pelo da Tommy Atkins. No que se refere ao ácido ascórbico e carotenóides totais, o resíduo desidratado da manga espada também apresentou os maiores teores (Tabelas 3 e 4). 
Figura 1. Capacidade de seqüestrar o Radical DPPH do extrato hidroacetônico (a); extrato hidrometanólico (b); extrato aquoso (c); mistura dos extratos hidroacetônico, hidrometanólico e aquoso (1:1:1, v/v/v) de manga Tommy Atkins; Rosa; Espada (300ppm) (d); do BHT e do ácido ascórbico (250ppm).

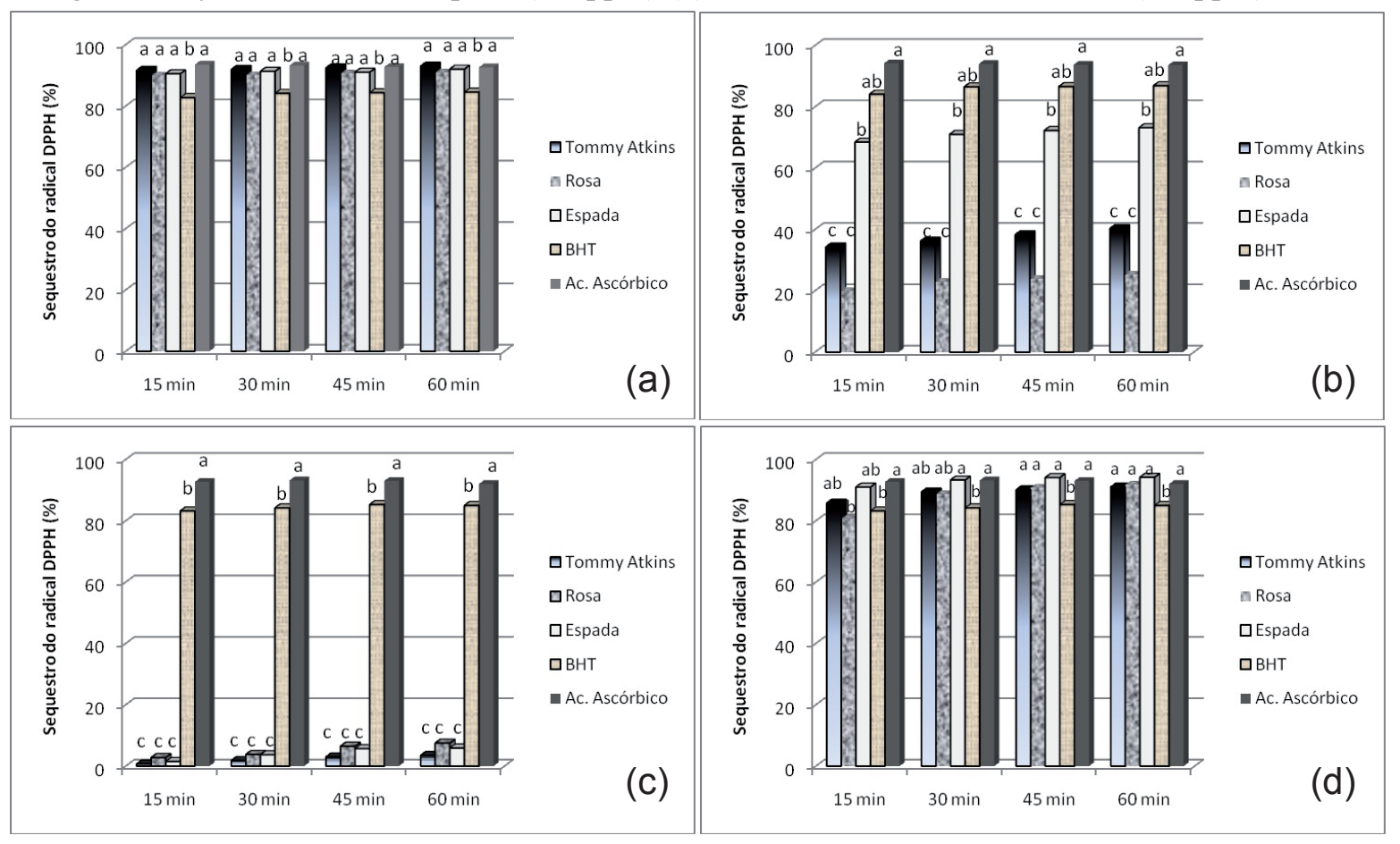

Tabela 3. Fenólicos totais* em extratos de resíduo desidratado de manga Espada, Rosa e Tommy Atkins.

\begin{tabular}{lccc}
\hline Manga & \multicolumn{3}{c}{ Extratos } \\
\cline { 2 - 4 } (variedades) & Hidroacetônico & Hidrometanólico & Total \\
\hline Espada & $2.828,27$ & 775,67 & $3.603,94$ \\
Rosa & 502,31 & 185,05 & 687,36 \\
Tommy Atkins & $1.305,02$ & 385,71 & $1.690,73$ \\
\hline
\end{tabular}

Médias de três determinações. Médias seguidas por letras iguais na coluna não difere de forma estatisticamente pelo Teste de Duncan $(\mathrm{p}<0,05) .{ }^{*} \mu \mathrm{g}$ em equivalente de catequina. $\mathrm{mL}^{-1}$ de extrato.

Tabela 4. Teor de fitoquímicos em resíduo desidratado de manga Espada, Rosa e Tommy Atkins.

\begin{tabular}{|c|c|c|c|}
\hline Resíduo desidratado de manga & $\begin{array}{l}\text { Fenólicos totais } \\
\left(\mathrm{mg.100g}^{-1}\right)\end{array}$ & $\begin{array}{c}\text { Ácido ascórbico } \\
\left(\mathbf{m g . 1 0 0 g ^ { - 1 } )}\right.\end{array}$ & $\begin{array}{c}\text { Carotenóides Totais } \\
\left(\mu \mathrm{g} \cdot \mathrm{g}^{-1}\right)\end{array}$ \\
\hline Espada & $3.603,94 \pm 28,69 a$ & $127,36 \pm 7,12 \mathrm{a}$ & $95,17 \pm 7,36 \mathrm{a}$ \\
\hline Tommy Atkins & $2.489,77 \pm 54,78 b$ & $99,94 \pm 6,82 b$ & $48,06 \pm 1,28 \mathrm{c}$ \\
\hline Rosa & $687,36 \pm 32,56 \mathrm{c}$ & $104,37 \pm 6,98 \mathrm{ab}$ & $85,2 \pm 6,25 b$ \\
\hline
\end{tabular}

Médias de três determinações independentes. Médias seguidas por letras iguais na linha não difere estatisticamente pelo Teste de Duncan $(\mathrm{p}<0,05)$. ${ }^{*} \mu \mathrm{g}$ em equivalente de $\beta$-caroteno. $\mathrm{g}^{-1}$ de amostra; $* *$ mg em equivalente de catequina. $100 \mathrm{~g}^{-1}$ 
O extrato hidroacetônico desses resíduos exibiu forte capacidade de seqüestro do radical $\mathrm{DPPH}$, superior a $80 \%$, não havendo diferença significativa entre os extratos das variedades de manga (Figura 2), enquanto que o hidrometanólico da manga Espada exibiu a mais elevada ação antioxidante (superior a 80\%), semelhante à exibida pelo ácido ascórbico e BHT. O extrato hidrometanólico do resíduo desidratado da manga Tommy Atkins exibiu capacidade de seqüestrar o radical DPPH superior a $60 \%$ e o da manga Rosa a mais baixa atividade (inferior a 50\%), quando comparada com a dos antioxidantes sintéticos (BHT e ácido ascórbico) e a do resíduo da manga Espada. Evidencia-se, portanto, que os resíduos de manga, independente da variedade exibe apreciável capacidade de seqüestrar o radical DPPH.

Figura 2. Capacidade de seqüestrar o Radical DPPH do extrato hidroacetônico (a) e do extrato hidrometanólico (b) de resíduo desidratado de manga Tommy Atkins, Rosa e Espada (300ppm); BHT e ácido ascórbico (250ppm).

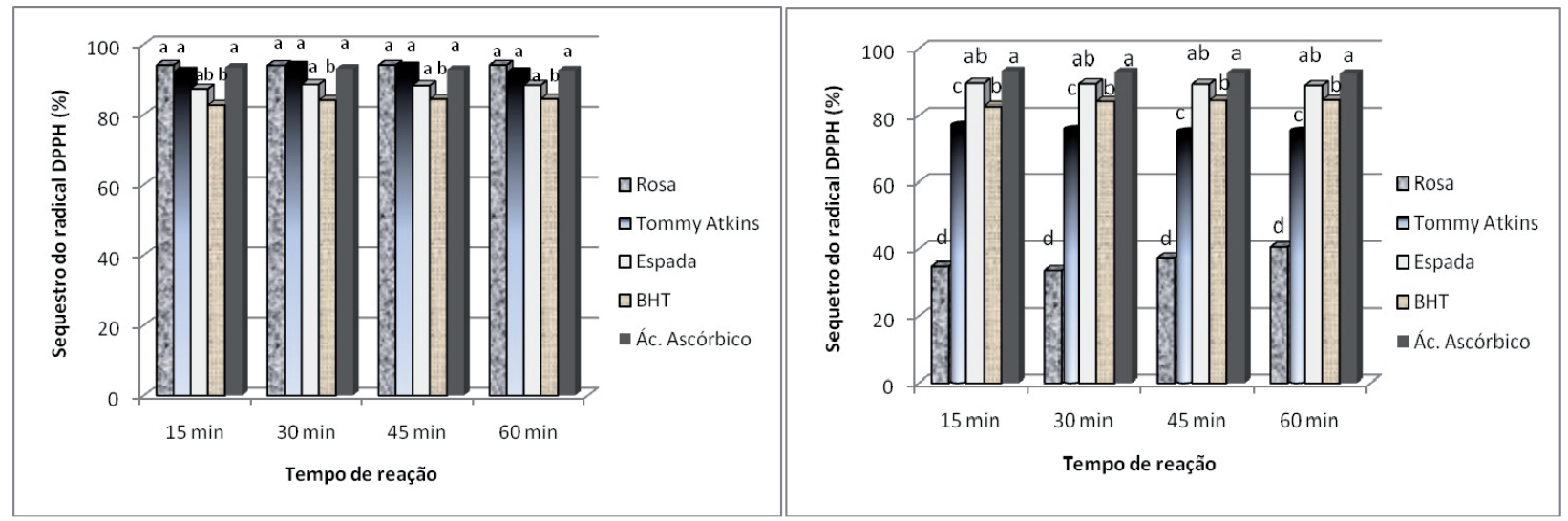

\section{Conclusão}

As mangas das variedades Espada, Rosa e Tommy Atkins possuem em sua constituição teor significante de ácido ascórbico, carotenóides e fenólicos totais, fitoquímicos com reconhecida ação antioxidante. A maioria dos compostos fenólicos das mangas das três variedades estudadas foi solubilizada em acetona a $80 \%$. Independente da variedade da manga, o extrato hidroacetônico exibiu a mais elevada capacidade de seqüestro do radical DPPH, revelando o forte potencial antioxidante da fruta. Frente ao teor de fitoquímicos e da capacidade de seqüestro do radical DPPH, as mangas das variedades Espada, Rosa e Tommy Atkins podem ser consideradas com uma importante fonte de antioxidante dietético, tornando importante sua participação na dieta usual .Os resíduos das três variedades de manga apresentaram teores consideráveis de fenólicos totais, cujos extratos exibiram forte capacidade de seqüestrar o radical DPPH, permitindo vislumbrar a possibilidade de empregá-los como aditivos em produtos alimentícios.

\section{Agradecimentos}

Ao $\mathrm{CNPq}$ (Edital MCT/CNPq 15/2007 -Universal) pelo auxílio financeiro.

\section{Referências}

ASSOCIATION OF OFFICIAL ANALYTICAL CHEMISTS - AOAC. Official methods of analysis of the association of official analytical chemists. $15^{\text {th }}$ ed. Arlington, V. A.: AOAC, 1990.

BEZERRA, T. S.; COSTA, J. M. C.; MAIA, G. A.; 
PINHO, L. X.; SANTOS, S. M. L. Avaliação físicoquímica de quatro variedades de manga comercializadas em Fortaleza-CE. In: CONGRESSO BRASILEIRO DE CIÊNCIA E TECNOLOGIA DOS ALIMENTOS, 21., 2008, Belo Horizonte. Anais ... Belo Horizonte. 2008. CD-ROM.

BENEVIDES, S. D.; RAMOS, A. M.; STRINGHETA, P. C.; CASTRO, V. C. Qualidade da manga e polpa da manga Ubá. Revista Ciência e Tecnologia de Alimentos, Campinas, v. 28, n. 3, p. 571-578, 2008.

BERNADES-SILVA, A. P. F.; LAJOLO, F. M.; CORDENUNSI, B. R. Evolução dos teores de amido e açúcares solúveis durante o desenvolvimento e amadurecimento de diferentes cultivares de manga. Ciência e Tecnologia de Alimentos, Campinas, v. 23, p.116-120, 2003. Suplemento.

BRANDÃO, M. C. C.; MAIA, G. A.; LIMA, D. P. Análise físico-química, microbiológica e sensorial de frutos de manga submetidos à desidratação osmóticosolar. Revista Brasileira de Fruticultura, Cruz das Almas, v. 25 , n. 1, p. 38-41, 2003.

BRAND-WILLIAMS, W.; CUVELIER, M.E.; BERSET, C. Use of free radical method to evaluate antioxidant activity. Lebensmittel Wissenschaft und Techonologie, London, v. 28, n. 1, p. 25-30, 1995.

CHEN, H. E.; TAI, C. Y.; CHEN, B. H. Improved liquid chromatographic method for determination of carotenoids in Taiwanese mango (Mangifera indica L.). Journal of Chromatography A, Washington, v. 1054, n. 1/2, p. 261-268, 2004

FRANKE, A. A.; CUSTER, L. J.; ARAKAKI, C.; MURPHY, S. P. Vitamin C and flavonoid levels of fruits and vegetables consumed in Hawaii. Journal of Food Composition and Analysis, Orlando, v. 17, n. 1, p. 1-35, 2004.

GENÚ, P. J.; PINTO, A. C. Q. A cultura da mangueira. Brasília: Embrapa Informação Tecnológica, 2002. 454 p.

GODOY, H. T.; RODRIGUEZ-AMAYA, D. B. Carotenoid composition of commercial mangoes from Brazil. Lebensmittel-Wissenschaft und-Technologie, London, v. 22, n. 3, p. 100-103, 1989.

GOMES, R. P. Fruticultura brasileira. 13. ed. São Paulo: Nobel, 1983. 446 p.

GUO, C.; YANG, J.; WEI, J.; LI, Y.; XU, J.; JIANG, Y. Antioxidant activities of peel, pul and seed fractions of common fruits as determined by FRAP assay. Nutrition Research, New York, v. 23, n. 12, p. 1719-1726, 2003.

INSTITUTO BRASILEIRO DE GEOGRAFIA E ESTATÍSTICA - IBGE. Disponível em: <http://www. sidra.ibge.gov.br>. Acesso em: 07 abr. 2009.

KAUR, C.; KAPOOR, H. C. Antioxidants in fruits and vegetables - the millennium's health. International Journal of Food Science and Technology, Oxford, v. 36, n. 7, p. 703-725, 2001.

KIM, Y.; BRECHT, J. K.; TALCOTT, S. T. Antioxidant phytochemical and fruit quality changes in mango (Mangifera indica L.) following hot water immersion and controlled atmosphere storage. Food Chemistry, Davis, v.105, n. 4, p. 1327-1334, 2007.

MELO, E. A.; LIMA, V. L. A. G.; MACIEL, M. I. S.; CAETANO, A. C. S.; LEAL, F. L. L. Polyphenol, Ascorbic acid and total carotenoid contents in common fruits and vegetables. Brazilian Journal of Food Technology, Campinas, v. 9, n. 2, p. 89-94, 2006.

MERCADANTE, A. Z. Estudo de carotenóides por espectrometria de massas e cromatografia líquida de alta eficiência. 1994. Tese (Doutorado em Ciência de Alimentos) - Universidade Estadual de Campinas, Campinas.

MILIAUSKAS, G.; VENSKUTONIS, P. R.; VAN BEEK, T. A. Screening of radical scavenging activity of some medicinal and aromatic plant extracts. Food Chemistry, Davis, v. 85, n. 2, p. 231-237, 2004.

NACZK, M.; SHAHIDI, F. Extraction and analysis of phenolics in food. Journal of Chromatography A, Washington, v. 1054, n. 1/2, p. 95-111, 2004.

PADILHA, M. R. F. Compostos bioativos em mangas (Mangifera indica L.): a influencia da cultivar, do estádio de maturação e do tratamento pós - colheita. 2005. Tese (Doutorado em Nutrição - Ciência dos Alimentos) Universidade Federal de Pernambuco, Recife.

PÉREZ-JIMÉNEZ, J.; ARRANZ, S.; TABERNERO, M.; DÍAZ-RUBIO, M. E.; SERRANO, J.; GONI, I.; SAURA-CALIXTO, F. Updated methodology to determine antioxidant capacity in plant, food, oils and beverages: extraction, measurement and expression of results. Food Research International, Canadá, v. 41, n. 3, p. 274-285, 2008.

RODRIGUEZ-AMAYA, D. B. A guide to carotenoids analysis in food. Washington: ILSI Press, 1999. 64 p.

SCALZO, J.; POLITI, A.; PELEGRINI, N.; MEZZETTI, B. M. Plant genotype affects total antioxidant capacity and phenolic contents in fruit. Nutrition, New York, v. 21, n.2, p. 207-213, 2005.

SILVA, E. M. F. Estudo sobre o mercado de frutas. São Paulo: FIPE, 1999. 373 p.

SOONG, Y-Y.; BARLOW, P. J. Antioxidant activity and phenolic content of selected fruit seeds. Food Chemistry, 
Davis, v. 88, n. 3, p. 411-417, 2004.

WETTASINGHE, M.; SHAHIDI, F. Evening primrose meal: a source of natural antioxidants and scavenger of hydrogen peroxide and oxygen-derived free radicals. Journal of Agricultural and Food Chemistry, Washington, v. 47, n. 5, p. 1801-1812, 1999. 
Melo, E. A.; Araújo, C. R. 